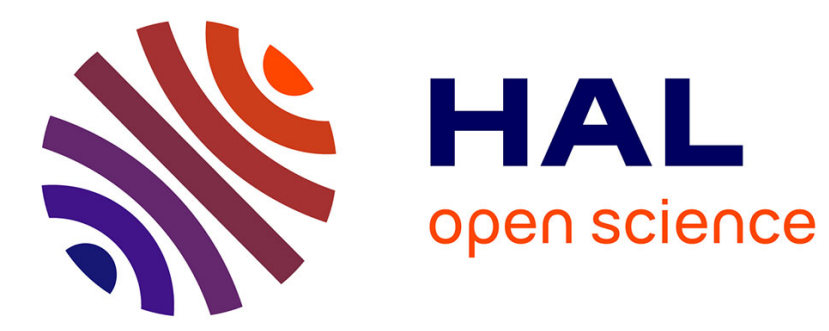

\title{
LONG TIME BEHAVIOUR OF SOLUTIONS TO AN EVOLUTION PDE WITH NONSTANDARD GROWTH
}

\author{
Hélène Frankowska, Gioconda Moscariello
}

\section{- To cite this version:}

Hélène Frankowska, Gioconda Moscariello. LONG TIME BEHAVIOUR OF SOLUTIONS TO AN EVOLUTION PDE WITH NONSTANDARD GROWTH. Advances in Calculus of Variation, 2020, 10.1515/acv-2019-0061 . hal-02906911

\section{HAL Id: hal-02906911 \\ https://hal.science/hal-02906911}

Submitted on 26 Jul 2020

HAL is a multi-disciplinary open access archive for the deposit and dissemination of scientific research documents, whether they are published or not. The documents may come from teaching and research institutions in France or abroad, or from public or private research centers.
L'archive ouverte pluridisciplinaire HAL, est destinée au dépôt et à la diffusion de documents scientifiques de niveau recherche, publiés ou non, émanant des établissements d'enseignement et de recherche français ou étrangers, des laboratoires publics ou privés. 


\title{
LONG TIME BEHAVIOUR OF SOLUTIONS TO AN EVOLUTION PDE WITH NONSTANDARD GROWTH
}

\author{
H. FRANKOWSKA AND G. MOSCARIELLO
}

\begin{abstract}
In this paper we prove time estimates for solutions to a general nonhomogeneous parabolic problem whose operator satisfies nonstandard growth conditions. We also study the asymptotic behaviour of solutons to an anisotropic problem.
\end{abstract}

Keywords. Evolution PDE, time dependence of solutions at infinity, generalized Gronwall lemma.

Mathematics Subject Classification. 35K51, 35K59, 35B40.

\section{INTRODUCTION.}

In this paper we investigate the time dependence of solutions for a wide class of nonuniformly parabolic equations whose model case is the following one:

$$
\begin{cases}u_{t}-\operatorname{div} \frac{G^{\prime}(|\nabla u|)}{|\nabla u|} \nabla u=0 & \text { in } \Omega_{T} \\ u(x, t)=0 & \text { on } \partial \Omega \times(0, T) \\ u(x, 0)=u_{0}(x) & \text { in } \Omega,\end{cases}
$$

where for some $N \geq 2, \Omega$ is a bounded domain in $\mathbb{R}^{N}$ with Lipschitz boundary $\partial \Omega, T$ is a positive number and $\Omega_{T}=\Omega \times(0, T)$.

We assume that $u_{0} \in L^{2}(\Omega)$ and $G: \mathbb{R}_{+} \rightarrow \mathbb{R}_{+}$is a $C^{1}$ convex function satisfying, for some $k>0$ and $p \geq 2$ the following conditions

$$
\begin{cases}(j) & G(0)=0 ; \quad G(2 s) \leq k G(s) \quad \forall s>0 \\ (j j) & \frac{G(s)}{s^{p}} \text { is increasing on }(0, \infty)\end{cases}
$$

We refer to $[16,26]$ for the properties of $G$ that follow from assumptions (1.2). The functions listed below do satisfy the above assumptions for $s \geq 0$ :

1) $G(s)=\frac{s^{p}}{p}, \quad p \geq 2$

2) $G(s)=s^{p} \log (1+s), \quad p \geq 2$;

3) $G(s)=s^{p} L_{k}(s), p \geq 2, L_{i}(s)=\log \left(1+L_{i-1}(s)\right), i=1, \ldots, k, L_{0}(s)=\log (1+s)$

4) $G(s)=\int_{0}^{s} g(\rho) d \rho$ where $g: \mathbb{R}_{+} \rightarrow \mathbb{R}_{+}$is a $C^{1}$ function satisfying

$$
p-1 \leq \frac{s g^{\prime}(s)}{g(s)} \leq q-1, \quad \forall s>0
$$

with $2 \leq p \leq q<\infty$ such that $\lim _{s \rightarrow+\infty} \frac{g(s)}{s}=+\infty$, see [3] where the properties of such a function $G$ were investigated. 
Definition 1.1. A function $u: \bar{\Omega}_{T} \rightarrow \mathbb{R}$ is called a weak solution to Problem (1.1) iff $u \in L^{\infty}\left(0, T ; L^{2}(\Omega)\right) \cap L^{1}\left(0, T ; W_{0}^{1,1}(\Omega)\right)$,

$$
\int_{0}^{T} \int_{\Omega} G(|\nabla u|) d x d t<+\infty
$$

and for every $\phi \in C^{1}\left(\bar{\Omega}_{T}\right)$ with $\left.\phi(\cdot, t)\right|_{\partial \Omega}=0$ the following equality holds

$$
\int_{\Omega} u \phi(x, t) d x-\int_{\Omega} u_{0} \phi(x, 0) d x+\int_{0}^{t} \int_{\Omega}\left[-u \phi_{s}+\left\langle\frac{G^{\prime}(|\nabla u|)}{|\nabla u|} \nabla u, \nabla \phi\right\rangle\right] d x d s=0
$$

for any $t \in[0, T]$.

If $u \in L_{\text {loc }}^{\infty}\left((0, \infty) ; L^{2}(\Omega)\right) \cap L_{\text {loc }}^{1}\left((0, \infty) ; W_{0}^{1,1}(\Omega)\right)$ and the above holds true for all $T>0$, then $u$ is called a weak solution to Problem $(1.1)$ on $(0, \infty) \times \Omega$.

In [8] the authors have shown that under (1.2) and some additional structural assumptions, there exists a unique weak solution $u \in C\left([0, T] ; L^{2}(\Omega)\right)$ to problem (1.1). When $G$ is as in example 4$)$, the existence and the uniqueness of a solution $u \in C\left(\bar{\Omega}_{T}\right)$ to a Cauchy-Dirichlet problem for evolution equation in (1.1) have been investigated in [3], again under several additional hypothesis.

In the elliptic framework equation in (1.1) is the Euler equation of the energy functional

$$
\int_{\Omega} G(|\nabla u|) d x
$$

where the convex integrand may have nonstandard growth conditions.

Note that the function $G$ in 2) satisfies for every $\epsilon>0$ the growth condition

$$
s^{p} \leq G(s) \leq L_{\epsilon}(1+s)^{p+\epsilon} .
$$

whenever $s$ is sufficiently large and where $L_{\epsilon}>0$ is a constant depending only on $\epsilon$ and $G$. Starting with the pioneering papers by P. Marcellini [19, 20], the theory in the stationary case, especially the regularity theory, has been extensively studied $([2,10,14,15,18,24$, $22,23])$. For an almost complete treatment see the survey [21] and the references therein. See also the recent paper [9].

Following the elliptic scheme, in [5] the authors recently studied variational solutions in the sense of [17] to the Cauchy Dirichlet problem (1.1). The existence of such solution has been proved in [7].

The objective of the present work is to obtain time estimates on weak solutions to (1.1) in terms of the $G$ function. More precisely, we get the following result:

Theorem 1.1. Assume (1.2) and let $u: \bar{\Omega}_{T} \rightarrow \mathbb{R}$ be a weak solution to problem (1.1). Then for any $t \in[0, T]$,

$$
\|u(\cdot, t)\|_{L^{2}(\Omega)}^{2} \leq 2|\Omega| x(t)
$$

where $x(\cdot)$ is the unique solution of the problem

$$
\left\{\begin{array}{l}
x^{\prime}(t)=-c G(\sqrt{x(t)}) \quad \text { a.e. } \\
x(0)=\frac{1}{2|\Omega|}\left\|u_{0}\right\|_{L^{2}(\Omega)}^{2}
\end{array}\right.
$$

and the constant $c>0$ depends only on $p, k,|\Omega|$.

Furthermore, if $T=\infty$, then $\lim _{t \rightarrow \infty}\|u(\cdot, t)\|_{L^{2}(\Omega)}^{2}=0$. 
Due to the nature of the problem, obtaining such estimates on solutions to (1.1) is not a trivial fact. As far as we know, Theorem 1.1 is the first result in this direction. Moreover, Theorem 1.1 implies time estimates also for evolution problems related to operators having standard growth conditions, as, for instance, for parabolic p-Laplace equation. Indeed Theorem 1.1 improves the results on the behaviour of a solution contained in [25] and in [12] (see Remark 4.2 below).

The main ingredient to achieve our result is a version of the Gronwall Lemma that has an interest by itself (see Lemma 3.1 and its two Corollaries in Section 3.). An energy balance equality for solutions to (1.1) (see Proposition 2.1) is also fundamental.

More generally, in this paper we prove time estimates, similar to those of Theorem 1.1 for solutions to a nonhomogeneous parabolic problem of the type

$$
\begin{cases}u_{t}-\operatorname{div} A(x, t, \nabla u)=-\operatorname{div} f & \text { in } \Omega_{T} \\ u(x, t)=0 & \text { on } \partial \Omega \times(0, T) \\ u(x, 0)=u_{0}(x) & \text { in } \Omega,\end{cases}
$$

where $A: \Omega_{T} \times \mathbb{R}^{N} \rightarrow \mathbb{R}^{N}$ is a Carathéodory function satisfying some nonstandard growth conditions and $f: \Omega_{T} \rightarrow \mathbb{R}^{N}$ satisfies the integrability condition (2.6) below.

Another application of our techniques is given in Section 5, where we study the asymptotic behaviour of solutions to an anisotropic problem.

The plan of the paper is as follows: In Section 2 we provide notations and preliminary results. In Section 3 we prove a generalized Gronwall Lemma. In Sections 4 and 5 we state and prove our main results.

\section{NotATION AND PRELIMINARY RESULTS}

Let us consider the parabolic problem

$$
\begin{cases}u_{t}-\operatorname{div} A(x, t, \nabla u)=-\operatorname{div} f & \text { in } \Omega_{T} \\ u(x, t)=0 & \text { on } \partial \Omega \times(0, T) \\ u(x, 0)=u_{0}(x) & \text { in } \Omega,\end{cases}
$$

where $\Omega$ is a Lipschitz bounded domain of $\mathbb{R}^{N}, N \geq 2, \Omega_{T}=\Omega \times(0, T)$ and $u_{0} \in L^{2}(\Omega)$. We assume that the field $A: \Omega_{T} \times \mathbb{R}^{N} \rightarrow \mathbb{R}^{N}$ is a Carathéodory function such that for a.e. $(x, t) \in \Omega_{T}$ and for any $\xi \in \mathbb{R}^{N}$

$$
\begin{cases}(i)\langle A(x, t, \xi), \xi\rangle \geq \nu G(|\xi|), & 0<\nu \leq 1 \\ (i i)|A(x, t, \xi)| \leq \mu \frac{G(|\xi|)}{|\xi|} & \xi \neq 0,1 \leq \mu \\ (\text { iii }) A(x, t, 0)=0, & \end{cases}
$$

where $G: \mathbb{R}_{+} \rightarrow \mathbb{R}_{+}$is a non zero convex function satisfying (1.2).

Let us remark that (2.2) could be imposed, equivalently, for $0<\nu<\mu$.

If $G$ is a $C^{1}$ non-trivial convex function satisfying $(\mathrm{j})$ in $(1.2)$, then the field $A(x, t, \xi)=$ $\frac{G^{\prime}(|\xi|)}{|\xi|} \xi, \xi \neq 0$, considered in the model case of Section 1 satisfies conditions (2.2). Indeed, in this case, since $G(0)=0$. The convexity of $G$ implies for all $\xi \in \mathbb{R}^{N}, \xi \neq 0$,

$$
G(|\xi|)=\int_{0}^{1} G^{\prime}(s|\xi|)|\xi| d s \leq\left\langle\frac{G^{\prime}(|\xi|)}{|\xi|} \xi, \xi\right\rangle
$$


and condition $(i)$ follows. Moreover, by the convexity of $G$ and from the second relation in $(j)$, we get for all $t>0$,

$$
k G(t) \geq G(2 t)=\int_{0}^{2 t} G^{\prime}(s) d s \geq \int_{t}^{2 t} G^{\prime}(s) d s \geq t G^{\prime}(t)
$$

from which $(i i)$ follows.

The Fenchel conjugate of $G$ is defined by

$$
\tilde{G}(r)=\sup _{s \geq 0}(r s-G(s))
$$

Then the Fenchel's inequality

$$
s r \leq G(s)+\tilde{G}(r)
$$

holds true for every $r, s \geq 0$. Note that $\tilde{G}: \mathbb{R} \rightarrow \mathbb{R}_{+}, \tilde{G}(0)=0$ and $\tilde{G}$ is increasing (in this paper we say increasing for nondecreasing).

Another useful property is (see [26] Chapt. II, (1))

$$
\tilde{G}\left(\frac{G(r)}{r}\right) \leq G(r) \quad \text { for every } r>0 .
$$

Observe that $G$ is increasing and it is not difficult to realise that unless $G$ is the zero function, $(j)$ implies that $G(s)>0$ for all $s>0$.

Condition $(j)$, which is known as the $\Delta_{2}$-condition, is equivalent to the assumption that there exists $q>1$ such that $\frac{G(t)}{t^{q}}$ is decreasing. On the other hand $(j j)$ is equivalent to $\Delta_{2}$ condition for the conjugate function $\tilde{G}$ of $G$, i.e. there exists $\tilde{k}>0$ such that $\tilde{G}(2 r) \leq \tilde{k} \tilde{G}(r)$ for every $r>0$ (see [16] Sect. 4). Consequently, $\tilde{G}(\cdot)>0$ on $(0, \infty)$.

Notice that $\Delta_{2}$ condition yields

$$
G(\lambda s) \leq \lambda^{k} G(s), \quad \tilde{G}(\lambda s) \leq \lambda^{\tilde{k}} \tilde{G}(s) \quad \forall s \geq 0, \lambda \geq 1 .
$$

For other properties of function $\mathrm{G}$ we refer to $[16,26]$.

In Problem (2.1) the datum $f: \Omega_{T} \rightarrow \mathbb{R}^{N}$ is assumed to be a measurable function such that

$$
\int_{0}^{T} \int_{\Omega} \tilde{G}(|f(x, s)|) d x d s<+\infty
$$

Definition 2.1. A function $u: \bar{\Omega}_{T} \rightarrow \mathbb{R}$ is a weak solution to Problem (2.1) iff

$$
\begin{gathered}
u \in L^{\infty}\left(0, T ; L^{2}(\Omega)\right) \cap L^{1}\left(0, T ; W_{0}^{1,1}(\Omega)\right) \\
\int_{0}^{T} \int_{\Omega}\langle A(x, s, \nabla u), \nabla u\rangle d x d s<+\infty
\end{gathered}
$$

and for every $\phi \in C^{1}\left(\bar{\Omega}_{T}\right)$ with $\left.\phi(\cdot, t)\right|_{\partial \Omega}=0$ the following equality holds

$$
\int_{\Omega} u \phi(x, t) d x-\int_{\Omega} u_{0} \phi(x, 0) d x+\int_{0}^{t} \int_{\Omega}\left[-u \phi_{s}+\langle A(x, s, \nabla u), \nabla \phi\rangle\right] d x d s=\int_{0}^{t} \int_{\Omega}\langle f, \nabla \phi\rangle d x d s
$$

for any $t \in[0, T]$. 
If $u \in L_{\text {loc }}^{\infty}\left((0, \infty) ; L^{2}(\Omega)\right) \cap L_{l o c}^{1}\left((0, \infty) ; W_{0}^{1,1}(\Omega)\right)$ and the above holds true for all $T>0$, then $u$ is called a weak solution to Problem $(2.1)$ on $(0, \infty) \times \Omega$.

Notice that assumption (2.8) is equivalent to the following condition

$$
\int_{0}^{T} \int_{\Omega} G(|\nabla u|) d x d t<+\infty .
$$

Indeed, since $\tilde{G}$ is increasing, by $(2.3),(i i),(2.4)$ and $(2.5)$, for any $(x, s) \in \Omega_{T}$ with $\nabla u(x, s) \neq 0$ we have

$$
\begin{aligned}
& |\langle A(x, s, \nabla u(x, s)), \nabla u(x)\rangle| \leq|A(x, s, \nabla u(x, s))| \cdot|\nabla u(x)| \\
& \leq \tilde{G}(|A(x, s, \nabla u(x, s))|)+G(|\nabla u(x)|) \leq \tilde{G}\left(\mu \frac{G(|\nabla u(x, s)|)}{|\nabla u(x, s)|}\right)+G(|\nabla u(x)|) \\
& \leq \mu^{\tilde{k}} \tilde{G}\left(\frac{G(|\nabla u(x, s)|)}{|\nabla u(x, s)|}\right)+G(|\nabla u(x)|) \leq \mu^{\tilde{k}} G(|\nabla u(x, s)|)+G(|\nabla u(x)|) .
\end{aligned}
$$

The equivalence between (2.8) and (2.10) follows from the first condition in (2.2) and from (2.11).

Remark 2.1. Using conditions (2.3), (2.6) and (2.10) respectively, an argument similar to $(2.11)$ proves that $\langle f(\cdot), \nabla \phi(\cdot)\rangle$ and $\langle A(\cdot, \cdot, \nabla u(\cdot, \cdot)), \nabla \phi(\cdot)\rangle$ are integrable on $\Omega_{T}$, for every $\phi \in C^{1}\left(\bar{\Omega}_{T}\right)$. These considerations imply that under our assumptions, all integrals in (2.9) are well defined.

Remark 2.2. By assumptions (1.2), for any $r>0$ we have

$$
\lambda_{1} r^{q^{\prime}} \leq \tilde{G}(r) \leq \lambda_{2}\left(r^{p^{\prime}}+1\right)
$$

for some positive constants $\lambda_{1}, \lambda_{2}$ and $p p^{\prime}=p+p^{\prime}$, and $q q^{\prime}=q+q^{\prime}$ (see [26] Chapt. 2). Then, from (2.9) we deduce that $u_{t} \in L^{q^{\prime}}\left(0, T ; L^{q^{\prime}}(\Omega)\right)$ with $1<q^{\prime}<2$, and so there exists $\tilde{u} \in C\left([0, T] ; L^{q^{\prime}}(\Omega)\right)$ such that $u(\cdot, t)=\tilde{u}(\cdot, t)$ for almost all $t \in(0, T)$. In what folllows we identify $u$ with its continuous representant $\tilde{u}$.

Lemma 2.1. Let $u \in L^{\infty}\left(0, T ; L^{2}(\Omega)\right) \cap L^{1}\left(0, T ; W_{0}^{1,1}(\Omega)\right)$, be a solution to Problem $(2.1)$. Then, $u \in C_{w}\left([0, T] ; L^{2}(\Omega)\right)$.

Proof. Let $t \in[0, T]$ be fixed. Let $\left\{t_{n}\right\}$ be a sequence in $[0, T]$ with $t_{n} \rightarrow t$ as $n \rightarrow+\infty$ such that $u\left(\cdot, t_{n}\right) \in L^{2}(\Omega) \forall n \in \mathbb{N}$. Since $u \in L^{\infty}\left(0, T ; L^{2}(\Omega)\right)$, the sequence $\left\{u\left(\cdot, t_{n}\right)\right\}$ is bounded in $L^{2}(\Omega)$. Then, there exists a subsequence $\left\{u\left(\cdot, t_{k_{n}}\right)\right\}$ and $\xi \in L^{2}(\Omega)$ such that

$$
u\left(\cdot, t_{k_{n}}\right) \rightarrow \xi \quad \text { weakly in } L^{2}(\Omega) \quad \text { as } n \rightarrow+\infty .
$$

On the other hand, by Remark $2.2 u \in C\left([0, T] ; L^{q^{\prime}}(\Omega)\right)$. Therefore

$$
u\left(\cdot, t_{k_{n}}\right) \rightarrow u(\cdot, t) \quad \text { strongy in } L^{q^{\prime}}(\Omega) \quad \text { as } n \rightarrow+\infty .
$$

This implies that $\xi=u(\cdot, t)$ and $u \in C_{w}\left([0, T] ; L^{2}(\Omega)\right)$.

We also recall the following useful result. 
Lemma 2.2. Let $G: \mathbb{R}_{+} \rightarrow \mathbb{R}_{+}$be a function satisfying (1.2). Then there exists a convex function $F: \mathbb{R}_{+} \rightarrow \mathbb{R}_{+}$satisfying $(j)$ such that

$$
F\left(t^{p}\right) \leq G(t) \leq k F\left(t^{p}\right) .
$$

where $k$ and $p$ are as in (1.2).

Proof. Since $G\left(s^{\frac{1}{p}}\right) / s$ is increasing, it is not difficult to realize that the function

$$
F(t)=\int_{0}^{t} \frac{G\left(s^{\frac{1}{p}}\right)}{s} d s
$$

is well defined on $(0, \infty)$ and can be extended by continuity to zero by setting $F(0)=0$. Its first derivative is a continuous increasing function $(0, \infty)$. Hence $F$ is convex on $\mathbb{R}_{+}$.

Moreover, by $(j j)$, the integrand is increasing and therefore

$$
F(t) \leq \int_{0}^{t} \frac{G\left(t^{\frac{1}{p}}\right)}{t} d s=G\left(t^{\frac{1}{p}}\right)
$$

implying the first inequality. We show next that $F$ satisfies $(j)$. Indeed, since $G$ satisfies $(j)$

$$
F(2 t)=\int_{0}^{t} \frac{G\left((2 s)^{\frac{1}{p}}\right)}{s} d s \leq \int_{0}^{t} \frac{G\left(2 s^{\frac{1}{p}}\right)}{s} d s \leq k \int_{0}^{t} \frac{G\left(s^{\frac{1}{p}}\right)}{s} d s=k F(t) .
$$

The second inequality in (2.12) follows from the following estimates

$$
G\left(t^{\frac{1}{p}}\right)=\int_{t}^{2 t} \frac{G\left(t^{\frac{1}{p}}\right)}{t} d s \leq \int_{t}^{2 t} \frac{G\left(s^{\frac{1}{p}}\right)}{s} d s \leq F(2 t) \leq k F(t) .
$$

In this paper we do not investigate the existence of a solution to $(2.1)$ and refer to $[3,7,8]$ for some existence results. Instead, we establish some properties of weak solutions. We first show that every such solution satisfies an energy balance equality.

Proposition 2.1. Assume (2.2) and (2.6) and let $u: \bar{\Omega}_{T} \rightarrow \mathbb{R}$ be a weak solution to (2.1). Then for any $0<t \leq T$

$$
\frac{1}{2}\|u(t)\|_{L^{2}(\Omega)}^{2}+\int_{0}^{t} \int_{\Omega}\langle A(x, s, \nabla u), \nabla u\rangle d x d s=\frac{1}{2}\left\|u_{0}\right\|_{L^{2}(\Omega)}^{2}+\int_{0}^{t} \int_{\Omega}\langle f(x, s), \nabla u\rangle d x d s .
$$

where $\|u(t)\|_{L^{2}(\Omega)}^{2}:=\int_{\Omega}|u(x, t)|^{2} d x$.

Proof. Let $u: \bar{\Omega}_{T} \rightarrow \mathbb{R}$ be a weak solution to (2.1). We first extend solution $u(x, t)$ by the initial value $u_{0}(x)$ when $t<0$. As $\partial \Omega$ is Lipschitz, we can cover $\partial \Omega$ by a finite open family $\left\{U_{i}\right\}_{i=1}^{m}$ and we can find corresponding positive numbers $\lambda_{i}$ and vectors $p_{i}$ such that for $\epsilon>0$ the ball $B\left(x+\lambda_{i} \epsilon p_{i}, \epsilon\right) \subset \Omega$ for all $x \in U_{i} \cap \Omega$ (cf. [11]).

Choose an open set $U_{0} \subset \subset \Omega$ such that $\left\{U_{i}\right\}_{i=0}^{m}$ forms a covering of $\Omega$ and let $\left\{\eta_{i}\right\}_{i=0}^{m}$ be a smooth partition of unity corresponding to this covering. For $x \in U_{i} \cap \Omega, i=1, \ldots, m$, denote $u_{i}^{\epsilon}(x, t)=u\left(x+\lambda_{i} \epsilon p_{i}, t\right)$ and let $u_{\epsilon}^{i}(\cdot, t)$ be its mollification in $U_{i} \cap \Omega$. Finally we mollify $u(x, t)$ in $U_{0}$ to get $u_{\epsilon}^{0}$. 
The functions $u_{\epsilon}: \bar{\Omega}_{T} \rightarrow \mathbb{R}$ defined as

$$
u_{\epsilon}=\sum_{i=0}^{m} \eta_{i} u_{\epsilon}^{i}
$$

are $C_{0}^{\infty}(\Omega)$ and converge to $u$ in $W_{0}^{1,1}(\Omega)$ when $\epsilon \rightarrow 0^{+}$for a.e. $t \in[0, T]$.

Consider the mollifier in time of $u_{\epsilon}(x, \cdot)$ defined for any natural $n \in \mathbb{N}$ such that $\frac{1}{n}<\epsilon$, as:

$$
\left[u_{\epsilon}\right]_{n}(x, t)=e^{-n t} u_{\epsilon}\left(x, t_{0}\right)+n \int_{0}^{t} e^{n(s-t)} u_{\epsilon}(x, s) d s
$$

where $t_{0} \in(0, T)$. Since $u_{\epsilon} \in L^{\infty}\left(0, T ; L^{2}(\Omega)\right) \cap L^{1}\left(0, T ; W_{0}^{1,1}(\Omega)\right)$ and $u_{\epsilon}\left(x, t_{0}\right) \in L^{2}(\Omega)$, we get that

$$
\begin{gathered}
{\left[u_{\epsilon}\right]_{n} \rightarrow u_{\epsilon} \text { in } L^{2}\left(\Omega_{T}\right) \text { as } n \rightarrow \infty .} \\
{\left[u_{\epsilon}\right]_{n}(x, 0)=u_{\epsilon}\left(x, t_{0}\right) \quad \forall n \in \mathbb{N} .} \\
{\left[u_{\epsilon}\right]_{n} \in L^{1}\left([0, T] ; W_{0}^{1,1}(\Omega)\right)} \\
\nabla\left[u_{\epsilon}\right]_{n}(\cdot, t)=\left[\nabla u_{\epsilon}\right]_{n}(\cdot, t) \\
{\left[\nabla u_{\epsilon}\right]_{n} \rightarrow \nabla u_{\epsilon} \text { in } L^{1}\left(\Omega_{T}\right) \text { when } n \rightarrow \infty .}
\end{gathered}
$$

Properties (2.13), (2.14) and (2.15) can be deduced from Lemma B2 in [5]. See also [6].

Now we define for any $\epsilon>0, n \in \mathbb{N}, 0<h<<1$ such that $0<\frac{1}{n}<\epsilon<h<t_{0}$, the Steklov average of $\left[u_{\epsilon}\right]_{n}$

$$
\phi_{\epsilon, h}^{n}(x, t):=\left(\left[u_{\epsilon}\right]_{n}\right)_{h}=\frac{1}{2 h} \int_{t-h}^{t+h}\left[u_{\epsilon}\right]_{n}(x, \tau) d \tau
$$

where we assume that $\left[u_{\epsilon}\right]_{n}(x, t)=u_{\epsilon}\left(x, t_{0}\right)$ for any $t \leq 0$.

First let us assume that $u$ is bounded. Then the function $\phi_{\epsilon, h}^{n} \in C^{1}\left(\bar{\Omega}_{T}\right)$ and $\phi_{\epsilon, h}^{n}(\cdot, t)_{\mid \partial \Omega}=$ 0 for every $t \in[0, T]$, and so we can use it as a test function in (2.9) to get that for any $t \in[0, T]$,

$$
\left.\int_{\Omega} u \phi_{\epsilon, h}^{n} d x\right|_{0} ^{t}+\int_{0}^{t} \int_{\Omega}\left[-u\left(\phi_{\epsilon, h}^{n}\right)_{s}+\left\langle A(x, s, \nabla u), \nabla \phi_{\epsilon, h}^{n}\right\rangle\right] d x d s=\int_{0}^{t} \int_{\Omega}\left\langle f, \nabla \phi_{\epsilon, h}^{n}\right\rangle d x d s .
$$

Set

$$
I_{1, n}^{\epsilon, h}:=\int_{\Omega} u \phi_{\epsilon, h}^{n}(x, t) d x-\int_{\Omega} u_{0} \phi_{\epsilon, h}^{n}(x, 0) d x-\int_{0}^{t} \int_{\Omega} u\left(\phi_{\epsilon, h}^{n}\right)_{s} d x d s .
$$

For $0<h<t$, by definition of $\phi_{\epsilon, h}^{n}$ 


$$
\begin{aligned}
I_{1, n}^{\epsilon, h} & =\int_{\Omega} u(x, t)\left(\frac{1}{2 h} \int_{t-h}^{t+h}\left[u_{\epsilon}\right]_{n}(x, \tau) d \tau\right) d x \\
& -\int_{\Omega} u_{0}(x)\left(\frac{1}{2 h} \int_{-h}^{h}\left[u_{\epsilon}\right]_{n}(x, \tau) d \tau\right) d x \\
& -\frac{1}{2 h} \int_{0}^{t} \int_{\Omega} u(x, \tau)\left(\left[u_{\epsilon}\right]_{n}(x, \tau+h)-\left[u_{\epsilon}\right]_{n}(x, \tau-h)\right) d x d \tau . \\
& =J_{1, n}^{\epsilon, h}+J_{2, n}^{\epsilon, h}+J_{3, n}^{\epsilon, h} .
\end{aligned}
$$

Observe that

$$
\begin{aligned}
J_{2, n}^{\epsilon, h}=-\int_{\Omega} u_{0}(x) & \left(\frac{1}{2 h} \int_{-h}^{0} u_{\epsilon}\left(x, t_{0}\right) d \tau+\frac{1}{2 h} \int_{0}^{h}\left[u_{\epsilon}\right]_{n}(x, \tau) d \tau\right) d x \\
J_{3, n}^{\epsilon, h} & =-\frac{1}{2 h} \int_{t}^{t+h} \int_{\Omega} u(x, \tau-h)\left[u_{\epsilon}\right]_{n}(x, \tau) d x d \tau \\
& -\frac{1}{2 h} \int_{h}^{t} \int_{\Omega} u(x, \tau-h)\left[u_{\epsilon}\right]_{n}(x, \tau) d x d \tau \\
& +\frac{1}{2 h} \int_{0}^{h} \int_{\Omega} u_{\epsilon}\left(x, t_{0}\right) u(x, \tau) d x d \tau \\
& +\frac{1}{2 h} \int_{h}^{t} \int_{\Omega}\left[u_{\epsilon}\right]_{n}(x, \tau-h) u(x, \tau) d x d \tau
\end{aligned}
$$

From (2.13), if we pass to the limit first as $n \rightarrow+\infty$ and then as $\epsilon \rightarrow 0^{+}$we get

$$
\begin{aligned}
\lim _{\epsilon \rightarrow 0+} & \lim _{n \rightarrow+\infty} I_{1, n}^{\epsilon, h}=\int_{\Omega} u(x, t)\left(\frac{1}{2 h} \int_{t-h}^{t+h} u(x, \tau) d \tau\right) d x \\
& -\frac{1}{2} \int_{\Omega} u_{0}(x) u\left(x, t_{0}\right) d x-\frac{1}{2 h} \int_{\Omega} u_{0}(x)\left(\int_{0}^{h} u(x, \tau) d \tau\right) d x \\
& -\frac{1}{2 h} \int_{t}^{t+h} \int_{\Omega} u(x, \tau-h) u(x, \tau) d x d \tau \\
& +\frac{1}{2 h} \int_{0}^{h} \int_{\Omega} u\left(x, t_{0}\right) u(x, \tau) d x d \tau .
\end{aligned}
$$

Then by Lemma 2.1, passing to the limit in $(2.16)$ as $h \rightarrow 0^{+}$, we get for any $t \in[0, T]$

$$
\lim _{h \rightarrow 0} \lim _{\epsilon \rightarrow 0} \lim _{n \rightarrow+\infty} I_{1, n}^{\epsilon, h}=\frac{1}{2} \int_{\Omega}|u(x, t)|^{2} d x-\frac{1}{2} \int_{\Omega}\left|u_{0}(x)\right|^{2} d x
$$

Define

$$
I_{2, n}^{\epsilon, h}:=\int_{0}^{t} \int_{\Omega}\left\langle A(x, s, \nabla u), \nabla \phi_{\epsilon, h}^{n}\right\rangle d x d s, \quad I_{3, n}^{\epsilon, h}:=\int_{0}^{t} \int_{\Omega}\left\langle f, \nabla \phi_{\epsilon, h}^{n}\right\rangle d x d s .
$$


Then,

$$
\begin{aligned}
I_{2, n}^{\epsilon, h} & =\sum_{i=0}^{m} \int_{0}^{t} \int_{\Omega}\left\langle A(x, s, \nabla u),\left(\left[u_{\epsilon}^{i}\right]_{n}\right)_{h}(x, s) \nabla \eta_{i}\right\rangle d x d s \\
& +\sum_{i=0}^{m} \int_{0}^{t} \int_{\Omega}\left\langle A(x, s, \nabla u), \eta_{i}(x)\left(\left[\nabla u_{\epsilon}^{i}\right]_{n}\right)_{h}(x, s)\right\rangle d x d s .
\end{aligned}
$$

Observe that

$$
\begin{aligned}
\left|\left\langle A(x, s, \nabla u), \eta_{i}(x)\left(\left[\nabla u_{\epsilon}^{i}\right]_{n}\right)_{h}(x, s)\right\rangle\right| & \leq \tilde{G}(|A(x, s, \nabla u)|)+G\left(\eta_{i}(x)\left|\left(\left[\nabla u_{\epsilon}^{i}\right]_{n}\right)_{h}\right|\right)(x, s) \\
& \leq \mu^{\tilde{k}} G(|\nabla u(x, s)|)+G\left(\eta_{i}(x)\left|\left(\left[\nabla u_{\epsilon}^{i}\right]_{n}\right)_{h}\right|\right)(x, s),
\end{aligned}
$$

where we used condition (ii) and inequalities (2.3), (2.4), (2.5).

Let $0<h \leq s$. By the Fubini theorem

$$
\begin{aligned}
& \left|\left(\left[\nabla u_{\epsilon}^{i}\right]_{n}\right)_{h}\right|(x, s) \leq \frac{1}{2 h} \int_{s-h}^{s+h}\left(e^{-n r}\left|\nabla u_{\epsilon}^{i}\left(x, t_{0}\right)\right|+n \int_{0}^{r} e^{n(\tau-r)}\left|\nabla u_{\epsilon}^{i}(x, \tau)\right| d \tau\right) d r \\
& =-\frac{1}{2 h n}\left[e^{-n(s+h)}-e^{-n(s-h)}\right]\left|\nabla u_{\epsilon}^{i}\left(x, t_{0}\right)\right|+\frac{n}{2 h} \int_{s-h}^{s+h}\left(\int_{\tau}^{s+h} e^{n(\tau-r)}\left|\nabla u_{\epsilon}^{i}(x, \tau)\right| d r\right) d \tau \\
& =\frac{1}{2 h n}\left[e^{-n(s-h)}-e^{-n(s+h)}\right]\left|\nabla u_{\epsilon}^{i}\left(x, t_{0}\right)\right|+\frac{1}{2 h} \int_{s-h}^{s+h}\left(1-e^{-n((s+h)-\tau)}\right)\left|\nabla u_{\epsilon}^{i}(x, \tau)\right| d \tau \\
& \leq \frac{1}{2 h n}\left|\nabla u_{\epsilon}^{i}\left(x, t_{0}\right)\right|+\frac{1}{2 h} \int_{s-h}^{s+h}\left|\nabla u_{\epsilon}^{i}(x, \tau)\right| d \tau .
\end{aligned}
$$

Inequality (2.18), the monotonicity of $G$ and the Jensen inequality imply that for $(x, s) \in$ $\Omega \times(h, t)$

$$
\begin{aligned}
& G\left(\eta_{i}\left|\left(\left[\nabla u_{\epsilon}^{i}\right]_{n}\right)_{h}\right|\right)(x, s) \leq G\left(\left|\left(\left[\nabla u_{\epsilon}^{i}\right]_{n}\right)_{h}\right|\right)(x, s) \\
& =G\left[\frac{1}{2 h n}\left|\nabla u_{\epsilon}^{i}\left(x, t_{0}\right)\right|+\frac{1}{2 h} \int_{s-h}^{s+h}\left|\nabla u_{\epsilon}^{i}(x, \tau)\right| d \tau\right] \\
& \leq \frac{1}{2} G\left(\frac{1}{h n}\left|\nabla u_{\epsilon}^{i}\left(x, t_{0}\right)\right|\right)+\frac{1}{2} G\left(2 \frac{1}{2 h} \int_{s-h}^{s+h}\left|\nabla u_{\epsilon}^{i}(x, \tau)\right| d \tau\right) \\
& \leq \frac{1}{2} G\left(\frac{1}{h n}\left|\nabla u_{\epsilon}^{i}\left(x, t_{0}\right)\right|\right)+2^{k-1} \frac{1}{2 h} \int_{s-h}^{s+h} G\left(\left|\nabla u_{\epsilon}^{i}(x, \tau)\right|\right) d \tau .
\end{aligned}
$$


When $0<s<h$, again by the Fubini theorem, we get

$$
\begin{aligned}
& \left|\left(\left[\nabla u_{\epsilon}^{i}\right]_{n}\right)_{h}\right|(x, s) \leq \frac{1}{2 h} \int_{s-h}^{0}\left|\nabla u_{\epsilon}^{i}\left(x, t_{0}\right)\right| d r \\
& +\frac{1}{2 h} \int_{0}^{s+h}\left(e^{-n r}\left|\nabla u_{\epsilon}^{i}\left(x, t_{0}\right)\right|+n \int_{0}^{r} e^{n(\tau-r)}\left|\nabla u_{\epsilon}^{i}(x, \tau)\right| d \tau\right) d r \\
& =\frac{h-s}{2 h}\left|\nabla u_{\epsilon}^{i}\left(x, t_{0}\right)\right|+\frac{1}{2 h n}\left[1-e^{-n(s+h)}\right]\left|\nabla u_{\epsilon}^{i}\left(x, t_{0}\right)\right| \\
& +\frac{n}{2 h} \int_{0}^{s+h}\left(\int_{\tau}^{s+h} e^{n(\tau-r)}\left|\nabla u_{\epsilon}^{i}(x, \tau)\right| d r\right) d \tau \\
& \leq\left(\frac{1}{2}+\frac{1}{2 h n}\right)\left|\nabla u_{\epsilon}^{i}\left(x, t_{0}\right)\right|+\frac{1}{2 h} \int_{0}^{s+h}\left(1-e^{-n((s+h)-\tau)}\right)\left|\nabla u_{\epsilon}^{i}(x, \tau)\right| d \tau \\
& \leq\left(\frac{1}{2}+\frac{1}{2 h n}\right)\left|\nabla u_{\epsilon}^{i}\left(x, t_{0}\right)\right|+\frac{1}{2 h} \int_{s-h}^{s+h}\left|\nabla u_{\epsilon}^{i}(x, \tau)\right| d \tau
\end{aligned}
$$

Arguing as in (2.19), from (2.20) we get, for $(x, s) \in \Omega \times(0, h)$

$$
\begin{aligned}
& G\left(\eta_{i}\left|\left(\left[\nabla u_{\epsilon}^{i}\right]_{n}\right)_{h}\right|\right)(x, s) \\
& \leq \frac{1}{2} G\left(\left(1+\frac{1}{h n}\right)\left|\nabla u_{\epsilon}^{i}\left(x, t_{0}\right)\right|\right)+2^{k-1} \frac{1}{2 h} \int_{s-h}^{s+h} G\left(\left|\nabla u_{\epsilon}^{i}(x, \tau)\right|\right) d \tau .
\end{aligned}
$$

Moreover, by the definition of $u_{\epsilon, h}^{i}$, the Fubini theorem and the Jensen inequality, we know that

$$
\left[G\left(\left|\nabla u_{\epsilon}^{i}\right|\right)\right]_{h}(x, s) \leq[G(|\nabla u|)]_{\epsilon, h}(x, s)
$$

Combining (2.17), (2.19) and (2.22), for almost every $(x, s) \in \Omega \times(h, t)$,

$$
\begin{aligned}
& \left|\left\langle A(x, s, \nabla u(x, s)),\left(\left[\nabla u_{\epsilon}^{i}\right]_{n}\right)_{h}(x, s) \eta_{i}(x)\right\rangle\right| \\
& \left.\leq \mu^{\tilde{k}} G(\mid \nabla u(x, s)) \mid\right)+\frac{1}{2} G\left(\frac{1}{h n}\left|\nabla u_{\epsilon}^{i}\left(x, t_{0}\right)\right|\right)+2^{k-1}[G(|\nabla u|)]_{\epsilon, h}(x, s)
\end{aligned}
$$

On the other hand, combining (2.17), (2.21) and (2.22), for almost every $(x, s) \in$ $\Omega \times(0, h)$,

$$
\begin{aligned}
& \left|\left\langle A(x, s, \nabla u(x, s)), \eta_{i}(x)\left(\left[\nabla u_{\epsilon}^{i}\right]_{n}\right)_{h}(x, s)\right\rangle\right| \\
& \left.\leq \mu^{\tilde{k}} G(\mid \nabla u(x, s)) \mid\right)+\frac{1}{2} G\left(\left(1+\frac{1}{h n}\right)\left|\nabla u_{\epsilon}^{i}\left(x, t_{0}\right)\right|\right)+2^{k-1}[G(|\nabla u|)]_{\epsilon, h}(x, s)
\end{aligned}
$$


Last inequalities, the continuity of $G$ and the Lebesgue dominated convergence theorem imply that

and

$$
\begin{gathered}
\lim _{h \rightarrow 0+} \lim _{\epsilon \rightarrow 0+} \lim _{n \rightarrow \infty} \int_{h}^{t} \int_{\Omega}\left\langle A(x, s, \nabla u(x, s)), \eta_{i}(x)\left(\left[\nabla u_{\epsilon}^{i}\right]_{n}\right)_{h}(x, s)\right\rangle d x d s \\
=\int_{0}^{t} \int_{\Omega}\left\langle A(x, s, \nabla u(x, s)), \eta_{i}(x) \nabla u(x, s)\right\rangle d x d s
\end{gathered}
$$

$$
\lim _{h \rightarrow 0+} \lim _{\epsilon \rightarrow 0+} \lim _{n \rightarrow \infty} \int_{0}^{h} \int_{\Omega}\left\langle A(x, s, \nabla u(x, s)), \eta_{i}(x)\left(\left[\nabla u_{\epsilon}^{i}\right]_{n}\right)_{h}(x, s)\right\rangle d x d s=0 .
$$

Finally, observe that

$\sum_{i=0}^{m} \int_{0}^{t} \int_{\Omega}\left\langle A(x, s, \nabla u(x, s)), \nabla u(x, s) \eta_{i}(x)\right\rangle d x d s=\int_{0}^{t} \int_{\Omega}\langle A(x, s, \nabla u(x, s)), \nabla u(x, s)\rangle d x d s$.

Consequently,

$$
\begin{aligned}
& \lim _{h \rightarrow 0+} \lim _{\epsilon \rightarrow 0+} \lim _{n \rightarrow \infty} \sum_{i=0}^{m} \int_{0}^{t} \int_{\Omega}\left\langle A(x, s, \nabla u(x, s)), \eta_{i}(x)\left(\left[\nabla u_{\epsilon}^{i}\right]_{n}\right)_{h}(x, s)(x)\right\rangle d x d s \\
& =\int_{0}^{t} \int_{\Omega}\langle A(x, s, \nabla u(x, s)), \nabla u(x, s)\rangle d x d s .
\end{aligned}
$$

On the other hand, since $\tilde{G}$ is increasing, by the Fenchel inequality, (ii), (2.4), (2.5), $(2.13)$ and the boundedness of $u$, for some $c_{0}>0$, for any $i \in\{0,1, \ldots, m\}$ and every $(x, s) \in \Omega_{T}$ with $\nabla u(x, s) \neq 0$ we have

$$
\begin{aligned}
& \left|\left\langle A(x, s, \nabla u(x, s)),\left(\left[u_{\epsilon}^{i}\right]_{n}\right)_{h}(x, s) \nabla \eta_{i}\right\rangle\right| \leq|A(x, s, \nabla u(x, s))| \cdot\left|\left(\left[u_{\epsilon}^{i}\right]_{n}\right)_{h}(x, s) \nabla \eta_{i}\right| \\
& \leq \tilde{G}(|A(x, s, \nabla u(x, s))|)+G\left(\left|\left(\left[u_{\epsilon}^{i}\right]_{n}\right)_{h}(x, s) \nabla \eta_{i}\right|\right) \leq \tilde{G}\left(\mu \frac{G(|\nabla u(x, s)|)}{|\nabla u(x, s)|}\right)+c_{0} \\
& \leq \mu^{\tilde{k}} \tilde{G}\left(\frac{G(|\nabla u(x, s)|)}{|\nabla u(x, s)|}\right)+c_{0} \leq \mu^{\tilde{k}} G(|\nabla u(x, s)|)+c_{0} .
\end{aligned}
$$

Hence $(2.10)$ implies that $\left|\left\langle A(\cdot, \cdot, \nabla u(\cdot, \cdot)),\left(\left[u_{\epsilon}^{i}\right]_{n}(\cdot, \cdot)\right)_{h} \nabla \eta_{i}(x)\right\rangle\right|$ is integrably bounded by a function not depending on $i, \epsilon, n, h$.

Therefore

Finally, observe that

$$
\begin{gathered}
\lim _{h \rightarrow 0+} \lim _{\epsilon \rightarrow 0+} \lim _{n \rightarrow \infty} \int_{0}^{t} \int_{\Omega}\left\langle A(x, s, \nabla u(x, s)),\left(\left[u_{\epsilon}^{i}\right]_{n}(x, s)\right)_{h} \nabla \eta_{i}(x)\right\rangle d x d s \\
=\int_{0}^{t} \int_{\Omega}\left\langle A(x, s, \nabla u(x, s)), u(x, s) \nabla \eta_{i}\right\rangle d x d s .
\end{gathered}
$$

$$
\sum_{i=0}^{m} \int_{0}^{t} \int_{\Omega}\left\langle A(x, s, \nabla u(x, s)), u(x, s) \nabla \eta_{i}(x)\right\rangle d x d s=0 .
$$

Consequently,

$$
\lim _{h \rightarrow 0+} \lim _{\epsilon \rightarrow 0+} \lim _{n \rightarrow \infty} \sum_{i=0}^{m} \int_{0}^{t} \int_{\Omega}\left\langle A(x, s, \nabla u(x, s)),\left(\left[u_{\epsilon}^{i}\right]_{n}\right)_{h}(x, s) \nabla \eta_{i}(x)\right\rangle d x d s=0 .
$$


From (2.23) and (2.25) we get

$$
\lim _{h \rightarrow 0+} \lim _{\epsilon \rightarrow 0+} \lim _{n \rightarrow \infty} I_{2, n}^{\epsilon, h}=\int_{0}^{t} \int_{\Omega}\langle A(x, s, \nabla u), \nabla u\rangle d x d s .
$$

In the same way, by using (2.3), (2.5) and (2.6), we can prove that

$$
\lim _{h \rightarrow 0+} \lim _{\epsilon \rightarrow 0+} \lim _{n \rightarrow \infty} I_{3, n}^{\epsilon, h}=\int_{0}^{t} \int_{\Omega}\langle f, \nabla u\rangle d x d s .
$$

If $u$ is not bounded, define for any $j \in \mathbb{N}, u_{j}=\frac{1}{2}(|u+j|-|u-j|)$. Then,

$$
u_{j} \in L^{\infty}\left(0, T ; L^{2}(\Omega)\right) \cap L^{1}\left(0, T ; W_{0}^{1,1}(\Omega)\right) \cap L^{\infty}\left(\Omega_{T}\right) .
$$

We can constuct $\left(u_{j}\right)_{\epsilon}$ as before and we can apply the above arguments by using $\left(\phi_{j}\right)_{\epsilon, h}^{n}$ as test function in (2.9) Finally we can pass to the limit as $j \rightarrow+\infty$ since $\left|u_{j}\right| \leq|u|$ a.e.

The uniqueness of a weak solution follows under the additional monotonicity condition

$$
\langle A(x, s, \xi)-A(x, s, \eta), \xi-\eta\rangle \geq 0
$$

for a.e. $(x, s) \in \Omega_{T}$ and for any $\xi, \eta \in \mathbb{R}^{n}$.

Proposition 2.2. Assume (2.2), (2.6) and (2.26). Let $u: \bar{\Omega}_{T} \rightarrow \mathbb{R}$ be a weak solution to (2.1). Then $u$ is unique.

Proof. The proof proceeds as in the homogeneous case. Let $v: \bar{\Omega}_{T} \rightarrow \mathbb{R}$ be a weak solution to (2.1). Apart of the approximation argument as in Proposition 2.1, we can use $w=u-v$ as test function in the definition of solutions $u$ and $v$.

Then, by subtracting we get for any $0<t \leq T$

$$
\frac{1}{2}\|w(t)\|_{L^{2}(\Omega)}^{2}+\int_{0}^{t} \int_{\Omega}\langle A(x, s, \nabla u)-A(x, s, \nabla v), \nabla w\rangle d x d s=0 .
$$

Hence, (2.26) yields $u=v$ a.e. in $\Omega_{T}$.

\section{A Version of the Gronwall Lemma}

For arbitrary non-empty closed sets in $D^{\prime}, D \subset \mathbb{R}^{n}$, define

$$
d_{D}\left(D^{\prime}\right):=\inf \left\{\beta>0 \mid D^{\prime} \subset D+\beta \mathcal{B}\right\} .
$$

where $B$ denotes the closed unit ball in $\mathbb{R}^{n}$. Consider reals $0 \leq t_{0}<T$ and a multifunction $P:\left[t_{0}, T\right] \leadsto \mathbb{R}^{n}$ with non-empty closed values. $P$ is called left absolutely continuous if the following condition is satisfied: given any $\epsilon>0$ there exists $\delta>0$ such that, for any finite partition

$$
t_{0} \leq s_{1}<\tau_{1} \leq s_{2}<\tau_{2} \leq \ldots \leq s_{m}<\tau_{m} \leq T
$$

satisfying $\sum_{i=1}^{m}\left(\tau_{i}-s_{i}\right)<\delta$, we have

$$
\sum_{i=1}^{m} d_{P\left(\tau_{i}\right)}\left(P\left(s_{i}\right)\right)<\epsilon .
$$


For any $y \in P(t)$ the contingent derivative $D P(t, y)(1)$ is the set of all $v \in \mathbb{R}^{n}$ such that there exist $h_{i} \rightarrow 0+$ and $v_{i} \rightarrow v$ satisfying $y+h_{i} v_{i} \in P\left(t+h_{i}\right)$.

A function $\gamma:\left[t_{0}, T\right] \rightarrow \mathbb{R}_{+}$is called upper semicontinuous from the right, if for every $t \in\left[t_{0}, T\right)$ we have $\lim \sup _{s \rightarrow t+} \gamma(s) \leq \gamma(t)$. Similarly, it is called lower semicontinuous from the left, if for every $t \in\left(t_{0}, T\right]$ we have $\liminf _{s \rightarrow t-} \gamma(s) \geq \gamma(t)$.

Lemma 3.1. Consider a Carathéodory function $\psi:\left[t_{0}, T\right] \times \mathbb{R} \rightarrow \mathbb{R}_{+}$such that for every $r>0$ there exists $k_{r} \in L^{1}\left(t_{0}, T ; \mathbb{R}_{+}\right)$satisfying for a.e. $t \in\left[t_{0}, T\right]$

$$
\sup _{|x| \leq r} \psi(t, x) \leq k_{r}(t) \text {. }
$$

Let $g \in L^{1}\left(t_{0}, T ; \mathbb{R}\right)$ and $\gamma:\left[t_{0}, T\right] \rightarrow \mathbb{R}_{+}$be measurable, bounded and satisfy

$$
\gamma\left(t_{2}\right)-\gamma\left(t_{1}\right)+\int_{t_{1}}^{t_{2}} \psi(t, \gamma(t)) d t \leq \int_{t_{1}}^{t_{2}} g(t) d t \quad t_{0} \leq t_{1} \leq t_{2} \leq T .
$$

Then there exists a solution $x(\cdot) \in W^{1,1}\left(\left[t_{0}, T\right]\right)$ of the Cauchy problem

$$
\left\{\begin{array}{l}
x^{\prime}(t)=-\psi(t, x(t))+g(t), \quad \text { a.e. } \\
x\left(t_{0}\right)=\gamma\left(t_{0}\right)
\end{array}\right.
$$

such that $\gamma(t) \leq x(t)$ for all $t \in\left[t_{0}, T\right]$.

Furthermore, if $g \in L^{1}\left(t_{0}, \infty ; \mathbb{R}\right), \psi$ is defined on $\left[t_{0},+\infty\right) \times \mathbb{R}, \gamma:\left[t_{0},+\infty\right) \rightarrow \mathbb{R}_{+}$ is measurable and locally bounded and for every $T>t_{0}$ the above assumptions hold true, then there exists a solution $x$ to (3.2) defined on $\left[t_{0}, \infty\right)$ such that $\gamma \leq x$. In particular,

$$
\limsup _{t \rightarrow \infty} \gamma(t) \leq \limsup _{t \rightarrow \infty} x(t) \text {. }
$$

Proof. By (3.1), $\gamma$ is upper semicontinuous from the right and lower semicontinuous from the left. Define the set-valued map $P:\left[t_{0}, T\right] \leadsto \mathbb{R}$ by

$$
P(t)= \begin{cases}\gamma(t)+\mathbb{R}_{+} & t \in\left[t_{0}, T\right) \\ \mathbb{R} & t \geq T .\end{cases}
$$

By our assumptions for all $t_{0} \leq t_{i}<\tau_{i}<T$

$$
\gamma\left(\tau_{i}\right)-\int_{t_{i}}^{\tau_{i}} g(t) d t \leq \gamma\left(t_{i}\right) .
$$

This implies that $P(\cdot)$ is left absolutely continuous. Let $t \in\left(t_{0}, T\right)$ be a Lebesgue point of $\psi(\cdot, \gamma(\cdot))$ and of $g(\cdot)$. The set $A$ of all such points is of full Lebesgue measure in $\left[t_{0}, T\right]$. By the assumptions of our lemma for every $t \in A$,

$$
D \gamma(t):=\liminf _{\varepsilon \rightarrow 0+} \frac{\gamma(t+\varepsilon)-\gamma(t)}{\varepsilon} \leq-\psi(t, \gamma(t))+g(t) .
$$

This and the definition of $P(\cdot)$ imply that $-\psi(t, \gamma(t))+g(t) \in D P(t, \gamma(t))(1)$. Since $\gamma$ is upper semicontinuous from the right, it enjoys the following property: if $y>\gamma(t)$, then for every $r \in \mathbb{R}$ and all small $\varepsilon>0$ we have $y+\varepsilon r>\gamma(t+\varepsilon)$. Therefore, $D P(t, y)(1)=\mathbb{R}$ for any $y>\gamma(t)$. Consequently for all $t \in A$ and $y \in P(t)$ we have $-\psi(t, y)+g(t) \in$ $D P(t, y)(1)$. By [13, Theorem 4.2] and our assumptions, for some $S \in\left(t_{0}, T\right]$ there exists a solution of (3.2) satisfying $x(t) \in P(t)$ on $\left[t_{0}, S\right)$. Therefore $0 \leq \gamma(t) \leq x(t)$ for all $t \in\left[t_{0}, S\right)$. Furthermore, $x(t) \leq x\left(t_{0}\right)+\int_{t_{0}}^{t} g(s) d s$ for all $t \in\left[t_{0}, S\right)$ implying 
that $x(\cdot)$ is bounded. Hence, by our assumptions and since $x(\cdot)$ is a solution of $(3.2)$, it can be extended, by continuity, on $\left[t_{0}, S\right]$. Furthermore, $\gamma(S) \leq x(S)$ because $\gamma$ is lower semicontinuous from the left. Applying the Zorn lemma and [13, Theorem 4.2], we extend this solution $x(\cdot)$ to the time interval $\left[t_{0}, T\right]$.

To prove the last statement assume that $g \in L^{1}\left(t_{0}, \infty ; \mathbb{R}\right), \psi$ is defined on $\left[t_{0},+\infty\right) \times \mathbb{R}$, $\gamma$ is locally bounded on $\left[t_{0},+\infty\right)$ and for every $T>t_{0}$ the assumptions of Lemma hold true. Then the same construction allows one to get $x(\cdot)$ defined on $\left[t_{0},+\infty\right)$ satisfying for every $t>\tau \geq t_{0}$,

$$
0 \leq \gamma(t) \leq x(t) \leq x(\tau)+\int_{\tau}^{t} g(s) d s .
$$

Since $\lim _{\tau \rightarrow \infty} \int_{\tau}^{\infty} g(s) d s=0$ the proof follows.

Remark 3.1. Indeed, assume that the solution $x:\left[t_{0}, T\right] \rightarrow \mathbb{R}_{+}$of (3.2) is unique. Then for $\gamma=x$ we have

$$
\gamma\left(t_{2}\right)-\gamma\left(t_{1}\right)+\int_{t_{1}}^{t_{2}} \psi(t, \gamma(t)) d t=\int_{t_{1}}^{t_{2}} g(t) d t \quad t_{0} \leq t_{1} \leq t_{2} \leq T .
$$

That is (3.1) holds true. The estimate $\gamma \leq x$ obtained in Lemma 3.1 can not be further improved.

Corollary 3.1. Under all the assumptions of Lemma 3.1 suppose that $\psi(t, a)=0$ for all $a \leq 0, g(\cdot) \geq 0, \psi(t, \cdot)$ is increasing for a.e. $t \in\left[t_{0}, T\right]$ and that for any $R>r>0$ there exists $\bar{k}_{R, r} \in L^{1}\left(t_{0}, T ; \mathbb{R}_{+}\right)$satisfying for a.e. $t \in\left[t_{0}, T\right]$

$$
|\psi(t, x)-\psi(t, y)| \leq k_{R, r}(t)|x-y| \quad \forall x, y \in[r, R] .
$$

Then the solution $z(\cdot)$ of

$$
\left\{\begin{array}{l}
z^{\prime}(t)=-\psi(t, z(t)), \quad \text { a.e. } \\
z\left(t_{0}\right)=\gamma\left(t_{0}\right)
\end{array}\right.
$$

is unique and well defined on $\left[t_{0}, T\right], z(\cdot) \geq 0$ and $\gamma(t) \leq x(t) \leq z(t)+\int_{t_{0}}^{t} g(s) d s$ for all $t \in\left[t_{0}, T\right]$.

Proof. Clearly, if $z(\cdot)$ is a solution of (3.3), then it is decreasing on its interval of existence. Moreover, if for some $t \geq t_{0}, z(t)=0$, then $z(s)=0$ for any $t \leq s \leq T$. This and the Lipschitz continuity assumption imply the existence and uniqueness on the whole time interval $\left[t_{0}, T\right]$. Consider any solution $x(\cdot)$ of $(3.2)$ defined on $\left[t_{0}, T\right]$. Then it is not difficult to realize that $x(\cdot) \geq 0$.

We claim that $z \leq x$ on $\left[t_{0}, T\right]$. Indeed otherwise we can find $t_{0} \leq s_{0}<t_{1}<T$ such that $z\left(s_{0}\right)=x\left(s_{0}\right)$ and $z(s)>x(s)$ on $\left[s_{0}, t_{1}\right]$. Then $-\psi(t, z(t)) \leq-\psi(t, x(t))$ for a.e. $t \in\left[s_{0}, t_{1}\right]$. Thus $x^{\prime}(s)-z^{\prime}(s) \geq g(s) \geq 0$ a.e. in $\left[s_{0}, t_{1}\right]$. This implies that $x(t) \geq z(t)$ for $t \in\left[s_{0}, t_{1}\right]$ leading to a contradiction. Let $x(\cdot)$ be as in Lemma 3.1. Observe that for a.e. $t \in\left[t_{0}, T\right]$ we have

$$
x^{\prime}(t)=-\psi(t, x(t))+g(t) \leq-\psi(t, z(t))+g(t)=z^{\prime}(t)+g(t) .
$$

Integrating we obtain that $x(t) \leq z(t)+\int_{t_{0}}^{t} g(s) d s$ for all $t \in\left[t_{0}, T\right]$. This and Lemma 3.1 yield $\gamma(t) \leq x(t) \leq z(t)+\int_{t_{0}}^{t} g(s) d s$. 
Corollary 3.2. Consider $g \in L^{1}\left(t_{0}, \infty ; \mathbb{R}_{+}\right)$, a Carathéodory function $\psi:\left[t_{0}, \infty\right) \times \mathbb{R} \rightarrow$ $\mathbb{R}_{+}$, a measurable locally bounded $\gamma:\left[t_{0}, \infty\right) \rightarrow \mathbb{R}_{+}$and let assumptions of Corollary 3.1 hold true on $\left[t_{0}, T\right]$ for every $T>t_{0}$. If for every $r>0$ there exists $\alpha_{r}>0$ such that $\inf _{x \geq r} \psi(t, x) \geq \alpha_{r}$ for a.e. $t \geq t_{0}$, then $\lim _{t \rightarrow \infty} \gamma(t)=0$.

Proof. Fix any sequence $t_{i} \rightarrow \infty$ and consider the solutions $z_{i}$ to (3.3) with $t_{0}$ replaced by $t_{i}$. By Corollary 3.1 applied with $t_{0}$ replaced by $t_{i}$, we know that $z_{i} \geq 0$. Notice that $z_{i}$ is decreasing for every $i$. Observe next that if for some $i$ we have $\lim _{t \rightarrow \infty} z_{i}(t)=: r>0$, then, $z_{i}(t) \geq r$ for every $t \geq t_{i}$ and

$$
0 \leq z_{i}(t)=z_{i}\left(t_{i}\right)-\int_{t_{i}}^{t} \psi\left(s, z_{i}(s)\right) d s \leq z_{i}\left(t_{i}\right)-\left(t-t_{i}\right) \alpha_{r} .
$$

When $t$ is sufficiently large, the right-hand side of the above expression becomes negative. Thus implies that $\lim _{t \rightarrow \infty} z_{i}(t)=0$ for all $i$. This and Corollary 3.1 imply that for every $i$, every $\varepsilon>0$ and all large $t$ we have

$$
\gamma(t) \leq \frac{\varepsilon}{2}+\int_{t_{i}}^{t} g(s) d s .
$$

Taking $i$ sufficiently large, we deduce that for every $\varepsilon>0$ and all large $t$ we have $\gamma(t) \leq \varepsilon$ completing the proof.

In [25] a particular instance of $\psi(t, x)$ was considered, namely $\psi(t, x)=M|x|^{1+\nu}$, where $M$ and $\nu$ are non negative constants. It was shown then that for any continuous $\gamma$ : $\left[t_{0}, T\right] \rightarrow \mathbb{R}_{+}$satisfying the inequality (3.1) we have

$$
\gamma(t) \leq y(t):=\frac{\gamma\left(t_{0}\right)}{\left[1+\nu M \gamma\left(t_{0}\right)^{\nu}\left(t-t_{0}\right)\right]^{\frac{1}{\nu}}}+\Gamma(t) \quad \forall t \in\left[t_{0}, T\right]
$$

for every choice of continuous function $\Gamma:\left[t_{0}, T\right] \rightarrow \mathbb{R}_{+}$satisfying

$$
\Gamma\left(t_{1}\right) \leq \Gamma\left(t_{2}\right)+M \int_{t_{1}}^{t_{2}} \Gamma(t)^{1+\nu} d t-\int_{t_{1}}^{t_{2}} g(t) d t \quad t_{0} \leq t_{1} \leq t_{2} \leq T,
$$

where $g \in L^{1}\left(\left[t_{0}, T\right], \mathbb{R}\right)$. Let $\Gamma, M, \nu$ be as above and $x$ be the solution of

$$
\left\{\begin{array}{l}
x^{\prime}=-M|x|^{1+\nu}+g(t), \quad \text { a.e. } t>t_{0} \\
x\left(t_{0}\right)=\gamma\left(t_{0}\right)
\end{array}\right.
$$

We already know that $x(t) \geq \gamma(t) \geq 0$ for all $t \in\left[t_{0}, T\right]$.

We claim that $x(t) \leq y(t)$, which implies that our result provides a better estimate on the time behavior of $\gamma$ than the one from [25]. Indeed, since $y\left(t_{0}\right)=\gamma\left(t_{0}\right)+\Gamma\left(t_{0}\right) \geq x\left(t_{0}\right)$, if for some $t_{1} \in\left(t_{0}, T\right]$ we have $y\left(t_{1}\right)<x\left(t_{1}\right)$, then we can find $s_{0} \in\left[t_{0}, t_{1}\right]$ such that $x\left(s_{0}\right)=y\left(s_{0}\right)$ and $y(s)<x(s)$ for all $s \in\left(s_{0}, t_{1}\right]$.

Note that $-y(\cdot)$ is continuous on $\left[t_{0}, T\right)$. By the same arguments as in the proof of Lemma 3.1, we check that $t \leadsto-\Gamma(t)+\mathbb{R}_{+}$is left absolutely continuous on $\left[t_{0}, T\right]$ and that the map

$$
t \mapsto \frac{\gamma\left(t_{0}\right)}{\left[1+\nu M \gamma\left(t_{0}\right)^{\nu}\left(t-t_{0}\right)\right]^{\frac{1}{\nu}}}
$$


is Lipschitz. Therefore $t \leadsto P(t)=-y(t)+\mathbb{R}_{+}$is left absolutely continuous on $\left[t_{0}, T\right]$. Furthermore, for a.e. $s \in\left(s_{0}, t_{1}\right)$ we have

$$
D(-\Gamma)(s) \leq M \Gamma(s)^{1+\nu}-g(s) .
$$

Hence, for a.e. $s \in\left(s_{0}, t_{1}\right)$,

$$
\begin{gathered}
D(-y)(s) \leq \frac{M \gamma\left(t_{0}\right)^{1+\nu}}{\left[1+\nu M \gamma\left(t_{0}\right)^{\nu}\left(t-t_{0}\right)\right]^{\frac{1+\nu}{\nu}}}+M \Gamma(s)^{1+\nu}-g(s) \leq M y(s)^{1+\nu}-g(s) \\
=M y(s)^{1+\nu}-M x(s)^{1+\nu}-x^{\prime}(s) \leq-x^{\prime}(s) .
\end{gathered}
$$

Therefore, for a.e. $s \in\left[s_{0}, t_{1}\right]$ and all $z \in P(s)$ we have $-x^{\prime}(s) \in D P(s, z)(1)$. From [13, Theorem 4.2] applied to the single-valued map $s \mapsto\left\{-x^{\prime}(s)\right\}$ we deduce that $-x(t) \in P(t)$ for all $t \in\left[s_{0}, t_{1}\right]$. This yields $x(t) \leq y(t)$ on $\left[s_{0}, t_{1}\right]$ contradicting the choice of $t_{1}$.

It was observed in [25] that if $g \geq 0$, then $\Gamma(t)=\int_{t_{0}}^{t} g(s) d s$ verifies (3.5) and that for any continuous $\gamma:\left[t_{0}, T\right] \rightarrow \mathbb{R}_{+}$satisfying the inequality (3.1), the estimate (3.4) holds true for this choice of $\Gamma$. In particular, if $g \in L^{1}\left(t_{0}, \infty ; \mathbb{R}_{+}\right)$, then (3.4) is verified with this $\Gamma$ and $t_{0}$ replaced by any $\tau>t_{0}$ and $T>\tau$. This implies that $\lim _{t \rightarrow \infty} \gamma(t)=0$.

Define $\psi(t, x)=M|x|^{1+\nu}$ for $x \geq 0$ and $\psi(t, x)=0$ for $x<0$ and observe that it satisfies the assumptions of Corollary 3.2. Thus the equality $\lim _{t \rightarrow \infty} \gamma(t)=0$ is a consequence of Corollary 3.2.

\section{The MAIN ReSUlts}

In this section we assume that the function $G: \mathbb{R}_{+} \rightarrow \mathbb{R}_{+}$in (2.2) is continuous, convex and satisfies (1.2) for some $k>0$ and $p \geq 2$.

We state next the main result of this paper.

Theorem 4.1. Assume (2.2) and (2.6). Let $u: \bar{\Omega}_{T} \rightarrow \mathbb{R}$ be a weak solution to (2.1). Then, for any $t \in[0, T]$,

$$
\|u(\cdot, t)\|_{L^{2}(\Omega)}^{2} \leq 2|\Omega| x(t)
$$

where $x(\cdot)$ is the unique solution of the problem

$$
\left\{\begin{array}{l}
\dot{x}(t)=-c G(\sqrt{x(t)})+g(t) \\
x(0)=\frac{1}{2|\Omega|}\left\|u_{0}\right\|_{L^{2}(\Omega)}^{2}
\end{array}\right.
$$

for some $c>0$ depending only on $p, k, N,|\Omega|, \nu$ and $g(t)=\frac{1}{|\Omega|}\left\|\tilde{G}\left(\frac{2}{\nu}|f(\cdot, t)|\right)\right\|_{L^{1}(\Omega)}$.

If $f: \Omega \rightarrow \mathbb{R}$ is a integrable function on $\Omega$, we set

$$
f_{\Omega}|f| d x=\frac{1}{|\Omega|} \int_{\Omega}|f| d x
$$

where $|\Omega|>0$ denotes the Lebesgue measure of $\Omega$.

Proof. For $0<t_{1}<t_{2}<T$, applying Proposition 2.1 at times $t_{1}$ and $t_{2}$ and subtracting the results we get

$$
\frac{1}{2}\left\|u\left(t_{2}\right)\right\|_{L^{2}(\Omega)}^{2}-\frac{1}{2}\left\|u\left(t_{1}\right)\right\|_{L^{2}(\Omega)}^{2}+\int_{t_{1}}^{t_{2}} \int_{\Omega}\langle A(x, s, \nabla u), \nabla u\rangle d x d s=\int_{t_{1}}^{t_{2}} \int_{\Omega}\langle f, \nabla u\rangle d x d s .
$$


By $(i)$ and Fenchel's inequality (2.3) we get

$$
\frac{1}{2}\left\|u\left(t_{2}\right)\right\|_{L^{2}(\Omega)}^{2}-\frac{1}{2}\left\|u\left(t_{1}\right)\right\|_{L^{2}(\Omega)}^{2}+\frac{\nu}{2} \int_{t_{1}}^{t_{2}} \int_{\Omega} G(|\nabla u|) d x d s \leq \int_{t_{1}}^{t_{2}} \int_{\Omega} \tilde{G}\left(\frac{2}{\nu}|f(x, s)|\right) d x d s .
$$

Applying Hölder inequality we obtain, since $p \geq 2$,

$$
\left(f_{\Omega}|u|^{2} d x\right)^{\frac{p}{2}} \leq c f_{\Omega}|\nabla u|^{p} d x
$$

where $c>0$ depends only on $p, N,|\Omega|$.

Now, let $F$ be the convex function as in the claim of Lemma 2.2. Since $F$ is positive and $F(0)=0$, it is nondecreasing on $\mathbb{R}_{+}$. From (4.3) we deduce that

$$
F\left(\left(f_{\Omega}|u|^{2} d x\right)^{\frac{p}{2}}\right) \leq F\left(c f_{\Omega}|\nabla u|^{p} d x\right)
$$

In what follows $C$ denotes a positive constant depending only on $N, p, k,|\Omega|$, which may vary from line to line. From (2.5), (2.12), (4.4) and the Hölder and Jensen inequalities we deduce that for any $t \in[0, T]$,

$$
G\left(\left(\frac{1}{2} f_{\Omega}|u(x, t)|^{2} d x\right)^{\frac{1}{2}}\right) \leq G\left(\left(f_{\Omega}|u(x, t)|^{2} d x\right)^{\frac{1}{2}}\right) \leq C f_{\Omega} G(|\nabla u|) d x .
$$

Going back to (4.2), we deduce from (4.5) the following inequality

$$
\begin{aligned}
& \frac{1}{2} f_{\Omega}\left|u\left(x, t_{2}\right)\right|^{2} d x-\frac{1}{2} f_{\Omega}\left|u\left(x, t_{1}\right)\right|^{2} d x+\frac{\nu}{2 C} \int_{t_{1}}^{t_{2}} G\left[\left(\frac{1}{2} f_{\Omega}|u(x, t)|^{2} d x\right)^{1 / 2}\right] d t \\
& \leq \frac{1}{|\Omega|} \int_{t_{1}}^{t_{2}} \int_{\Omega} \tilde{G}\left(\frac{2}{\nu}|f(x, s)|\right) d x d s .
\end{aligned}
$$

Now we are in position to apply Lemma 3.1 with

$$
\gamma(t)=: \frac{1}{2} f_{\Omega}|u(x, t)|^{2} d x
$$

and

$$
\psi(t, x)=: \frac{\nu}{2 C} G(\sqrt{x}) .
$$

By the measurable viability theorem, cf. [13], there exists a solution $x(\cdot): \mathbb{R}_{+} \rightarrow \mathbb{R}_{+}$of (4.1). We claim that it is unique. Indeed, for positive reals $z \geq y$, we have $\sqrt{z} \geq \sqrt{y}$ and $\psi(t, z) \geq \psi(t, y)$. Hence $(\psi(t, z)-\psi(t, y))(z-y) \geq 0$. Similarly, it can be verified that if $z \leq y$, the same inequality holds true.

Consider any solution $y:\left[t_{0}, T\right] \rightarrow \mathbb{R}_{+}$of $(4.1)$. Then

$$
\frac{d}{d t} \frac{1}{2}|x-y|^{2}(t)=-(\psi(t, x(t))-\psi(t, y(t)))(x(t)-y(t)) \leq 0
$$

and therefore $x=y$ on $\left[t_{0}, T\right]$.

Setting $\psi(t, a)=0$ for $a<0$, we may apply Lemma 3.1 and so to get the desired estimate. 
Corollaries 3.1 and 3.2 yield the following result.

Theorem 4.2. In Theorem 4.1 assume that $g \in L^{1}([0, \infty))$. Let $u: \bar{\Omega} \times[0, \infty) \rightarrow \mathbb{R}$ be a weak solution to (2.1). Then, for any $t_{0} \geq 0$ and $t>t_{0}$,

$$
\|u(t, \cdot)\|_{L^{2}(\Omega)}^{2} \leq 2|\Omega| x(t) \leq 2|\Omega|\left(z(t)+\int_{t_{0}}^{t} g(s) d s\right)
$$

where $z(\cdot)$ is the unique solution of the problem

$$
\left\{\begin{array}{l}
\dot{z}(t)=-c G(\sqrt{z(t)}) \\
z\left(t_{0}\right)=\frac{1}{2|\Omega|}\left\|u\left(\cdot, t_{0}\right)\right\|_{L^{2}(\Omega)}
\end{array}\right.
$$

for some $c>0$ depending only on $p, k, N,|\Omega|, \nu$.

Furthermore, $\lim _{t \rightarrow \infty}\|u(t, \cdot)\|_{L^{2}(\Omega)}^{2}=0$.

Remark 4.1. Theorem 1.1 follows trivially from Theorem 4.1 and Theorem 4.2.

Remark 4.2. If $G(s)=\frac{s^{p}}{p}, p \geq 2$, as observed in Section 3, the statement of Theorem 4.1 improves the behaviour in time of a solution to Problem (2.1) for a p-Laplace type operator (see [25] and the reference therein). For istance, when $p=2$, Theorem 4.1 implies that for any $t \in[0, T]$

$$
\|u(t)\|_{L^{2}(\Omega)}^{2} \leq e^{-\frac{c}{2} t}\left\|u_{0}\right\|_{L^{2}(\Omega)}^{2}+2 \int_{0}^{t} e^{-\frac{c}{2}(t-s)}\|f(\cdot, s)\|_{L^{2}(\Omega)}^{2} d s
$$

where $c$ is the constant in Problem (4.1).

We conclude this section by an example.

Let us consider for simplicity problem (1.1) with

$$
G(s)=\left(s^{2}+1\right) \log \left(1+s^{2}\right) .
$$

Then, if $u$ is the weak solution of problem (1.1), we get that

$$
\|u(\cdot, t)\|_{L^{2}(\Omega}^{2} \leq 2|\Omega|\left(\exp \left(\log \left(1+\frac{1}{2|\Omega|}\left\|u_{0}\right\|_{L^{2}(\Omega)}\right) e^{-c t}\right)-1\right)
$$

for some $c>0$ and all $t \geq 0$.

Estimate (4.7) is new and cannot be achieved by the arguments of [25].

\section{Solutions of AN Anisotropic Problem}

In this section we consider the following anisotropic problem

$$
\left\{\begin{array}{l}
u_{t}-\sum_{i=1}^{N} \frac{\partial}{\partial x_{i}}\left(\left|u_{x_{i}}\right|{ }^{q_{i}-2} u_{x_{i}}\right)=0 \quad \text { in } \Omega_{T}, \\
u(x, t)=0 \quad \text { on } \partial \Omega \times(0, T), \\
u(x, 0)=u_{0}(x) \quad \text { in } \Omega,
\end{array}\right.
$$


where $u_{0} \in L^{2}(\Omega)$ and $1<q_{i}, i \in\{1, \ldots, N\}$.

Here, for any $\xi \in \mathbb{R}^{N}$ the vector field is $A(\xi)=: \sum_{i=1}^{N}\left|\xi_{i}\right|^{q_{i}-2} \xi_{i}$.

In [8] the authors prove that there exists a unique function $u \in C\left([0, T], L^{2}(\Omega)\right) \cap$ $L^{2}\left(0, T, W_{0}^{1,1}(\Omega)\right)$ with

$$
\int_{0}^{T} \int_{\Omega} \sum_{i=1}^{N}\left|u_{x_{i}}\right|^{q_{i}} d x d t<+\infty
$$

that solves (5.1). Moreover, for every $t \in(0, T]$ we have

$$
\frac{1}{2}\|u(t)\|_{L^{2}(\Omega)}^{2}+\int_{0}^{t} \int_{\Omega} \sum_{i=1}^{N}\left|u_{x_{i}}\right|^{q_{i}} d x d t=\frac{1}{2}\left\|u_{0}\right\|_{L^{2}(\Omega)}^{2} .
$$

The proof of (5.2) is similar to the one of Proposition 2.1. In this case the mollifier in time of test functions is not necessary and we can argue directly on the convex vector field $A(\xi)$. (See also [8]).

Set

$$
\begin{gathered}
\frac{1}{\bar{q}}=: \frac{1}{N} \sum_{i=1}^{N} \frac{1}{q_{i}}, \quad q=: \max \left\{q_{i}, i=1, \ldots, N\right\} \\
p=: \min \left\{q_{i}, i=1, \ldots, N\right\}
\end{gathered}
$$

and

$$
B(t)=: \max \left\{1,\left\|u_{x_{i}}(t)\right\|_{L^{q_{i}}(\Omega), i=1, \ldots, N}\right\}
$$

Theorem 5.1. Assume that $2 \leq q$ and $\frac{2 N}{N+2}<\bar{q}<N$. Let $u: \bar{\Omega}_{T} \rightarrow \mathbb{R}$ be a weak solution to problem (5.1). Then for any $t \in[0, T]$,

$$
\|u(\cdot, t)\|_{L^{2}(\Omega)}^{2} \leq 2 x(t)
$$

where $x(t)$ is the unique solution of the problem

$$
\left\{\begin{array}{l}
x^{\prime}(t)=-c B(t)^{p-q} x(t)^{\frac{q}{2}} \\
x(0)=\frac{1}{2}\left\|u_{0}\right\|_{L^{2}(\Omega)}^{2}
\end{array}\right.
$$

for some $c>0$ depending only on $|\Omega|, \bar{q}$ and $N$.

Proof. By (5.2) for any $0 \leq t_{1}<t_{2} \leq T$

$$
\frac{1}{2}\left\|u\left(t_{2}\right)\right\|_{L^{2}(\Omega)}^{2}-\frac{1}{2}\left\|u\left(t_{1}\right)\right\|_{L^{2}(\Omega)}^{2}+\int_{t_{1}}^{t_{2}} \int_{\Omega} \sum_{i=1}^{N}\left|u_{x_{i}}\right|^{q_{i}} d x d t=0
$$

We claim that, for a.e. $t \in(0, T)$,

$$
\left(\int_{\Omega}|u|^{\bar{q}^{*}} d x\right)^{\frac{q}{\bar{q}^{*}}} \leq c B(t)^{q-p} \int_{\Omega} \sum_{i=1}^{N}\left|u_{x_{i}}\right|^{q_{i}} d x
$$

where $c$ depends on $N,|\Omega|, \bar{q}$. 
In fact, by the definition of $B(t)$, since $q_{i} \geq p$ and $B(t) \geq 1$, for any $i=1, \ldots, N$ we have

$$
\left\|u_{x_{i}}\right\|_{L^{q_{i}(\Omega)}}^{q} \leq B(t)^{q-q_{i}}\left\|u_{x_{i}}\right\|_{L^{q_{i}(\Omega)}}^{q_{i}} \leq B(t)^{q-p}\left\|u_{x_{i}}\right\|_{L^{q_{i}(\Omega)}}^{q_{i}}
$$

The convexity of the real function $t \mapsto t^{q}$ implies

$$
\left(\frac{1}{N} \sum_{i=1}^{N}\left\|u_{x_{i}}\right\|_{L^{q_{i}(\Omega)}}\right)^{q} \leq \frac{1}{N} \sum_{i=1}^{N}\left\|u_{x_{i}}\right\|_{L^{q_{i}}(\Omega)}^{q}
$$

Then, since $\frac{2 N}{N+2}<\bar{q}<N$, we can apply the anisotropic Sobolev-type inequality [27] to obtain

$$
\|u\|_{L^{\bar{q}^{*}}(\Omega)}^{q} \leq c_{0}^{q}\left(\frac{1}{N} \sum_{i=1}^{N}\left\|u_{x_{i}}\right\|_{L^{q_{i}}(\Omega)}\right)^{q} \leq c_{0}^{q} N^{-1} B(t)^{q-p} \sum_{i=1}^{N}\left\|u_{x_{i}}\right\|_{L^{q_{i}}(\Omega)}^{q_{i}}
$$

where $c_{0}$ depends on $N,|\Omega|, \bar{q}$. Then, (5.5) follows.

The elliptic version of relation (5.5) has been proved in [4].

Now, from (5.5) we get

$$
B(t)^{p-q}\left(\int_{\Omega}|u|^{2} d x\right)^{\frac{q}{2}} \leq B(t)^{p-q}|\Omega|^{\frac{q}{2}-\frac{q}{\bar{q}^{*}}}\left(\int_{\Omega}|u|^{\bar{q}^{*}} d x\right)^{\frac{q}{\bar{q}^{*}}} \leq c_{1} \int_{\Omega} \sum_{i=1}^{N}\left|u_{x_{i}}\right|^{q_{i}} d x
$$

where $c_{1}$ depends on $N,|\Omega|, \bar{q}$.

Integrating (5.5) on $\left(t_{1}, t_{2}\right)$, from the relations (5.4) and (5.6) we get

$$
\frac{1}{2}\left\|u\left(t_{2}\right)\right\|_{L^{2}(\Omega)}^{2}-\frac{1}{2}\left\|u\left(t_{1}\right)\right\|_{L^{2}(\Omega)}^{2}+c \int_{t_{1}}^{t_{2}} B(t)^{p-q}\left(\frac{1}{2} \int_{\Omega}|u(x, t)|^{2} d x\right)^{\frac{q}{2}} d t \leq 0
$$

for some $c>0$ depending only on $N,|\Omega|, \bar{q}, q$. Now if $\gamma(t)=\frac{1}{2}\|u(t)\|_{L^{2}(\Omega)}^{2}$ we can rewrite (5.7) as

$$
\gamma\left(t_{2}\right)-\gamma\left(t_{1}\right)+\int_{t_{1}}^{t_{2}} \psi(t, \gamma(t)) d t \leq 0
$$

where

$$
\psi(t, x)=c B(t)^{p-q} x^{\frac{q}{2}} \forall x \geq 0, \quad \psi(t, x)=0 \quad \forall, x<0 .
$$

We apply Corollary 3.1 to achieve the announced result.

Remark 5.1. Let $\beta:[0, T] \rightarrow(1,+\infty)$ be a measurable function. The statement of Theorem 5.1 still holds if we replace the function $B(t), t \in[0, T]$, with the function

$$
\tilde{B}(t)=\max \left\{\left\|u_{x_{i}}(t)\right\|_{q_{i}}, \beta(t): i=1, \ldots, N\right\} .
$$

Indeed the above proof can be applied as well. 


\section{ACKNOWLEDGMENTS}

The authors are grateful to the referee for the constructive comments that helped to improve the presentation of the results. The research was partially supported by CNRS when G. Moscariello were visiting the center. Gioconda Moscariello was partially supported by Ministry of Education, University and Research PRIN 2017 and she is member of Gruppo Nazionale per l'Analisi Matematica, la Probabilità e le loro Applicazioni (GNAMPA) of INdAM.

\section{REFERENCES}

[1] Adams, R. Anisotropic Sobolev inequalities. Casopis Pest. Mat. 113 (1988), no. 3, 267-279.

[2] Acerbi, E., Mingione, G.: Regularity results for a class of functionals with non-standard growth. Arch. Ration. Mech. Anal. 156, 121-140 (2001) 3

[3] P. Baroni and C. Lindfors, The Cauchy-Dirichlet problem for a general class of parabolic equations. Ann. Inst. H. Poincaré Anal. Non Linéaire 34 (2017), no. 3, 593-624.

[4] L. Boccardo, P. Marcellini, and C. Sbordone: $L^{\infty}$-regularity for variational problems with sharp non-standard growth conditions. Boll. Unione Mat. Ital. VII. Ser. A 4 (1990), 219-225. Z

[5] V. Bögelein, F. Duzaar and P. Marcellini Parabolic systems with p,q-growth: a variational approach. Arch. Ration. Mech. Anal. 210 (2013), no. 1, 219-267

[6] Bögelein, Verena; Duzaar, Frank; Mingione, Giuseppe Degenerate problems with irregular obstacles. J. Reine Angew. Math. 650 (2011), 107-160.

[7] V. Bögelein, F. Duzaar and P. Marcellini, Existence of evolutionary variational solutions via the calculus of variations. J. Differential Equations 256 (2014), no. 12, 3912-3942.

[8] Y. Cai and S. Zhou Existence and uniqueness of weak solutions for a non-uniformly parabolic equation. J. Funct. Anal. 257 (2009), no. 10, 3021-3042.

[9] Cupini, G; Marcellini, P.; Mascolo, E., Nonuniformly elliptic energy integrals with p, q-growth. Nonlinear Anal. 177 (2018), part A, 312-324.

[10] L. Esposito, F. Leonetti, and G. Mingione: Higher integrability for minimizers of integral functionals with (p, q) growth. J. Differ. Equations 157 (1999), 414-438.

[11] L.C. Evans, Partial Differential Equations, Amer. Math, Soc. Providence, RI (1988)

[12] Farroni, F.; Moscariello, G. A nonlinear parabolic equation with drift term. Nonlinear Anal. 177 (2018), part B, 397-412.

[13] H. Frankowska, S. Plaskacz and T. Rzezuchowski, Measurable viability theorems and HamiltonJacobi-Bellman equation. Differential Equations 116 (1995), 265-305.

[14] N. Fusco, C. Sbordone: Higher integrability of the gradient of minimizers of functionals with nonstandard growth conditions. Commun. Pure Appl. Math. 43 (1990), 673-683

[15] N. Fusco, C. Sbordone: Some remarks on the regularity of minima of anisotropic integrals. Commun. Partial Differ. Equations 18 (1993), 153-167. Zbl 0795.49

[16] M. A. Krasnosel'skii and Ya.B. Rutitskii, Convex functions and Orlicz spaces, Noordhoff, Groningen, 1961.

[17] A. Lichnewsky and R. Temam, Pseudosolutions of the time-dependent minimal surface problem, J. Differential Equations 30 (1978), no. 3, 340-364.

[18] G. M. Lieberman: The natural generalization of the natural conditions of Ladyzhenskaya and Ural'tseva for elliptic equations. Commun. Partial Differ. Equations 16 (1991), 311-361.

[19] Marcellini, P: Regularity of minimizers of integrals of the calculus of variations with non standard growth conditions. Arch. Ration. Mech. Anal. 105, 267-284 (1989) 43.

[20] Marcellini, P.: Regularity and existence of solutions of elliptic equations with p, q-growth conditions. J. Differ. Equ. 90, 1-30 (1991) 44.

[21] Mingione G. Regularity of minima: an invitation to the dark side of the calculus of variations. Appl. Math. 51 (2006), no. 4, 355-426. 
[22] Moscariello, G. Local boundedness of minimizers of certain degenerate functionals of the calculus of variations. Nonlinear Anal. 23 (1994), no. 12, 1587-1593.

[23] Moscariello, G. Regularity results for quasiminima of functionals with nonpolynomial growth. J. Math. Anal. Appl. 168 (1992), no. 2, 500-510

[24] Moscariello, G. Nania L, Hölder continuity of minimizers of functionals with nonstandard growth conditions. Ric. Mat. 40 (1991), 259-273.

[25] Moscariello G:, Porzio, M.M. Quantitative asymptotic estimates for evolution problems. Nonlinear Analysis 154 (2017), 225-240.

[26] M. M. Rao and Z. Ren, Theory of Orlicz spaces. Monographs and Textbooks in Pure and Applied Mathematics, 146. Marcel Dekker, Inc., New York, 1991.

[27] Troisi M. Teoremi di inclusione per spazi di Sobolev non isotropi. Ricerche Mat. 18 (1969), 3-24.

CNRS, Institut de Mathématiques de Jussieu - Paris Rive Gauche,

Sorbonne Université, Case 247, 4 Place Jussieu, 75252 Paris, France

Email address: helene.frankowska@imj-prg.fr

Dipartimento di Matematica e Applicazioni, Università degli Studi di Napoli

"Federico II", Via Cintia, 80126 NAPOLI, ItAly

Email address: gmoscari@unina.it 